\title{
A unified homographic law for fusion excitation functions above the barrier
}

\author{
P. Eudes ${ }^{1, a}$, Z. Basrak ${ }^{2, b}$, F. Sébille ${ }^{1}$, V. de la Mota ${ }^{1}$, and G. Royer ${ }^{1}$ \\ ${ }^{1}$ SUBATECH, EMN-IN2P3/CNRS-Université de Nantes, Nantes, France \\ ${ }^{2}$ Ruđer Bošković Institute, Zagreb, Croatia
}

\begin{abstract}
We report on a systematics of fusion cross section data at energies above the reaction threshold to those of disappearance of fusion process. By an appropriate scaling of both cross sections and energy, a fusion excitation function common to all the data points is established. A universal description of the fusion excitation function relying on basic nuclear concepts is proposed and its dependence on the reaction cross section used for the cross section normalization is discussed.
\end{abstract}

\section{Introduction}

The evolution of the fusion reaction mechanism above the barrier to the Fermi-energy region is closely related to the existing competition between (nuclear) mean field and two-body dissipation. Thus, the study of both the complete (CF) and incomplete (IF) fusion excitation functions may be a proper way to understand and to constrain the ingredients entering in theoretical models, such as Sky3D [1], TDHF3D [2] or DYWAN [3], used to describe heavyion reactions at that energy range. Examples of burning issues are the choice of effective interactions [4] and the modeling of nucleon-nucleon collisions.

In two recent papers $[5,6]$ we have published a systematic study of both CF and IF fusion cross sections $\sigma_{\text {fus }}$ for incident energies $E_{\text {lab }} /$ nucleon $=E_{\text {in }}$ higher than about $3 A-4 A \mathrm{MeV}$. From the works published during the past 40 years we have collected $382 \mathrm{CF}$ and $\mathrm{CF}+\mathrm{IF} \sigma_{\text {fus }}$ data points belonging to 81 reaction systems with a vast variety of projectile-target pairs. By properly reducing $\sigma_{\text {fus }}$ values with the (total) reaction cross section $\sigma_{\text {reac }}$ and by applying an original energy scaling we have demonstrated that, irrespectively of the details of a given reaction system, the fusion excitation function follows a simple universal functional law. On that way we have been able to identify those data points for which, the most likely, the reported $\sigma_{\text {fus }}$ values suffer from a non-fusion contribution $[5,6]$ and those for which other reaction mechanism has been erroneously identified as fusion [7]. Discarding these data one ends with 76 systems and $316 \mathrm{CF}+\mathrm{IF} \sigma_{\text {fus }}$ data points plus 57 $\mathrm{CF}$ data points. Besides on experimental uncertainties of $\sigma_{\text {fus }}$ the exact parameter values of the universal functional law are somewhat dependent on the values of $\sigma_{\text {reac }}$ used for the $\sigma_{\text {fus }}$ normalization. In this contribution we focus on the data normalization problem and the impact of the implemented $\sigma_{\text {reac }}$.

\footnotetext{
ae-mail: eudes@subatech.in2p3.fr

be-mail: basrak@irb.hr
}

\section{Scaling of fusion cross section}

Figure 1 displays the $316 \mathrm{CF}+\mathrm{IF}$ kept $\sigma_{\text {fus }}$ data points as a function of $E_{\mathrm{in}}$. Clearly, most of these non-scaled raw $\sigma_{\text {fus }}$ data points gather in a narrow domain of the $\sigma_{\text {fus }}$ vs $E_{\text {in }}$ plane although lighter systems (blue, cyan, and green symbols) gather along an arclike structure decreasing with $E_{\text {in }}$ while heavier ones (pink, red, and orange symbols) follow a line which sharply rises with $E_{\text {in }}$.

The fact that systems of low and high mass do not fall together is natural due to the known dependence of $\sigma_{\text {fus }}$ on the system size. In order to compare so many different systems it is not enough to scale only the cross sections. We have shown $[5,6]$ that energy has to be scaled too. In order to express on the same footing the mass asymmetric systems with those which are mass symmetric the system energy should be expressed in units of the so-called system available energy that is nothing but the center-of-mass energy per nucleon

$$
E_{\text {avail }}=\frac{E_{\mathrm{c} . \mathrm{m} .}}{A_{\mathrm{tot}}}=\frac{E_{\mathrm{lab}}}{A_{\mathrm{p}}} \frac{A_{\mathrm{p}} A_{t}}{\left(A_{p}+A_{t}\right)^{2}},
$$

where $A_{\text {tot }}=A_{t}+A_{p}$ is the system mass and $A_{p}$ and $A_{t}$ are projectile and target mass, respectively.

One accounts for the proportionality of cross section to the size of a reaction system by the renormalizing $\sigma_{\text {fus }}$ by (total) reaction cross section $\sigma_{\text {reac }}$ at the same $E_{\mathrm{in}}$. Accurate measurement of the total $\sigma_{\text {reac }}$ is rather hard so that these data are scarce and it would be inappropriate to apply them to the ensemble of our $\sigma_{\text {fus }}$ data. Therefore, one commonly resorts to phenomenological approaches to calculate $\sigma_{\text {reac }}$, a solution which suffers for its own uncertainties and ambiguities. Thus, the uncertainty arising from the use of a particular parameterization of $\sigma_{\text {reac }}$ has to be investigated. Among a number of phenomenological parameterizations we have investigated five of them [8-12] including the pioneer one due to Bass [8] and the most recent one by Tripathi [12]. In order to infer reliability of 


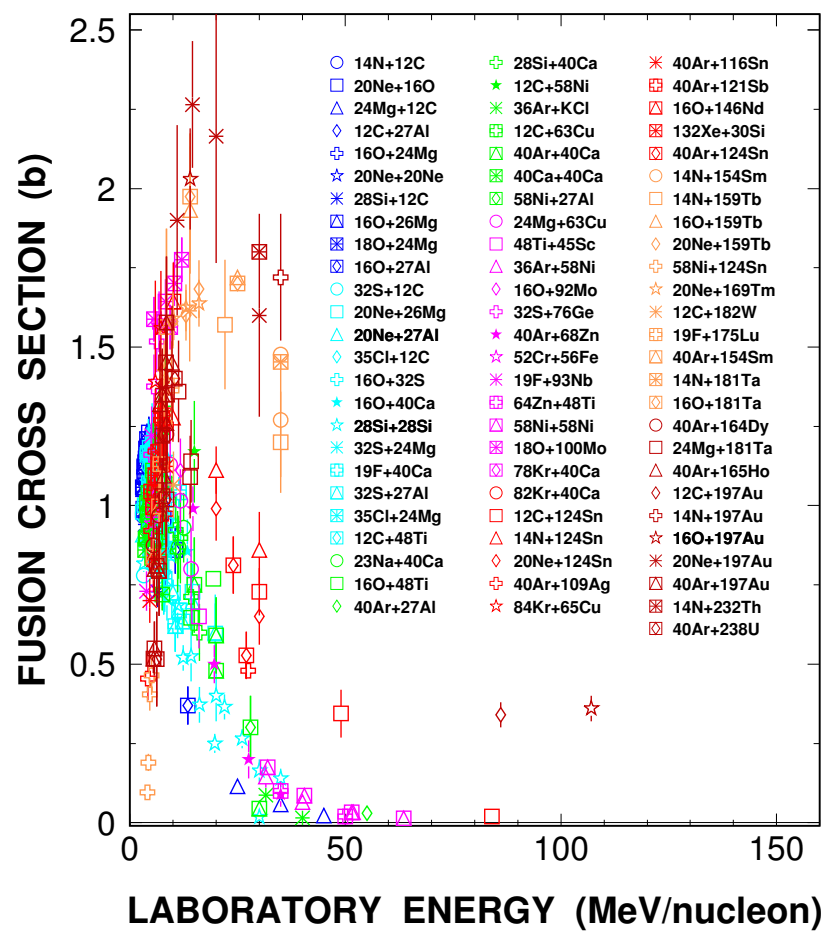

Figure 1. Raw fusion cross sections $\sigma_{\text {fus }}$ plotted as a function of $E_{\text {in }}$. The inventoried systems are distinguished among them by symbols and a color code. The same symbols and the color code are used in Ref. [6] where an interested reader may find detailed information on energies, $\sigma$ values, and references to original works.

each of the models in figure 2 we compare them with 134 experimental $\sigma_{\text {reac }}$ measured for 46 systems. All these approaches rely on the strong absorption picture of nuclear processes and differ among themselves in the way the basic relation

$$
\sigma(E)=\pi R^{2}\left(1-\frac{V}{E}\right)
$$

is parameterized. In Eq. (2) the cross section depends on the inverse of the (center-of-mass) energy $E$ while the radius $R$ and the potential depth $V$ may be, in a first approximation, considered constant for a given system. Various models differ in the treatment of $R$ and $V$ by introducing or not a certain dependence on energy and/or on some other system property, usually $A_{p}$ or $A_{\text {tot }}$, to the one or both of them.

From figure 2 one may conclude that the Bass approach overpredicts the measured cross sections at all $E_{\text {avail }}$, that the one of Kox underpredicts them at low energies ( $E_{\text {avail }} \lesssim 2 \mathrm{MeV} /$ nucleon) while that of Gupta at higher energies ( $E_{\text {avail }} \gtrsim 2 \mathrm{MeV} /$ nucleon). These conclusions are plausible from the histograms obtained by projecting those ratios on the ordinate of each of panels in figure 2 .

Figures 3 and 4 show the $\sigma_{\text {fus }}$ data of figure 1 scaled in both cross section and energy. In figure 3 shown are normalizations with $\sigma_{\text {reac }}$ of Bass, Gupta, and Kox, respectively and in figure 4 those with $\sigma_{\text {reac }}$ of Shen and that of Tripathi as well as the one labeled Mixed obtained by a heuristic approach. By investigating the projection histogram on the ordinate axis of the ratios between experi-

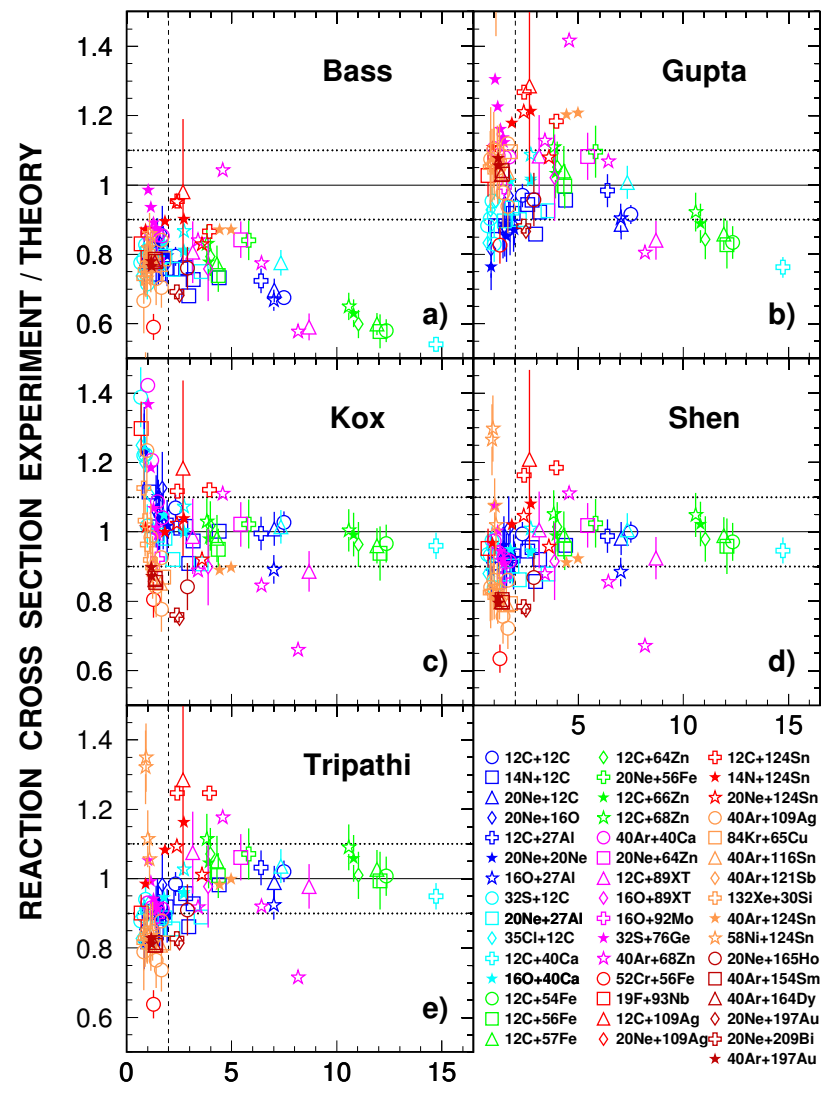

AVAILABLE ENERGY (MeV/nucleon)

Figure 2. Ratios of experimental reaction cross sections and theoretical predictions for five models as a function of $E_{\text {avail }}$. Panels display $\sigma_{\text {reac }}$ ratios with the models of a) Bass [8], b) Gupta and Kailas [9], c) Kox et al. [10], d) Shen et al. [11], and e) Tripathi et al. [12]. An interested reader may find detailed information on experimental $\sigma_{\text {reac }}$ and references to original works in Ref. [6].

mental and model reaction cross sections we heuristically define the most appropriate combination of model predictions. So, the Mixed $\sigma_{\text {reac }}$ is taken as that of Tripathi [12] for $E_{\text {avail }}<2 \mathrm{MeV} /$ nucleon and $A_{\text {tot }}<86$, as that of Gupta [9] for $E_{\text {avail }}<2 \mathrm{MeV} /$ nucleon and $A_{\text {tot }} \geq 86$, while for $E_{\text {avail }} \geq 2 \mathrm{MeV} /$ nucleon mixed $\sigma_{\text {reac }}$ is the average of the Shen [11] and Tripathi [12] predictions.

It is interesting to note that out of the 316 data points in the case of Gupta and that of Kox 2 and 41 points, respectively violate the physically allowed range for the normalized cross section values $\sigma_{\text {fus }} / \sigma_{\text {reac }}=\sigma^{\text {red }}$, namely between 0 and 1 . All these points are due to $\sigma^{\text {red }}$ overflow that occurs at low energies.

Assuming the applicability of the strong absorption concept in the first approximation both $\sigma_{\text {fus }}$ and $\sigma_{\text {reac }}$ may be expressed by the same functional form given by Eq. (2). Consequently, for the reduced cross section one gets a simple homographic law

$$
\sigma^{\mathrm{red}}(E)=\frac{\sigma_{\text {fus }}(E)}{\sigma_{\text {reac }}(E)}=a+\frac{b}{c+E},
$$

where $a, b$, and $c$ are free parameters and $E$ is taken as $E_{\text {avail }}[5,6]$. The $\sigma^{\text {red }}$ data points of each panel of fig- 


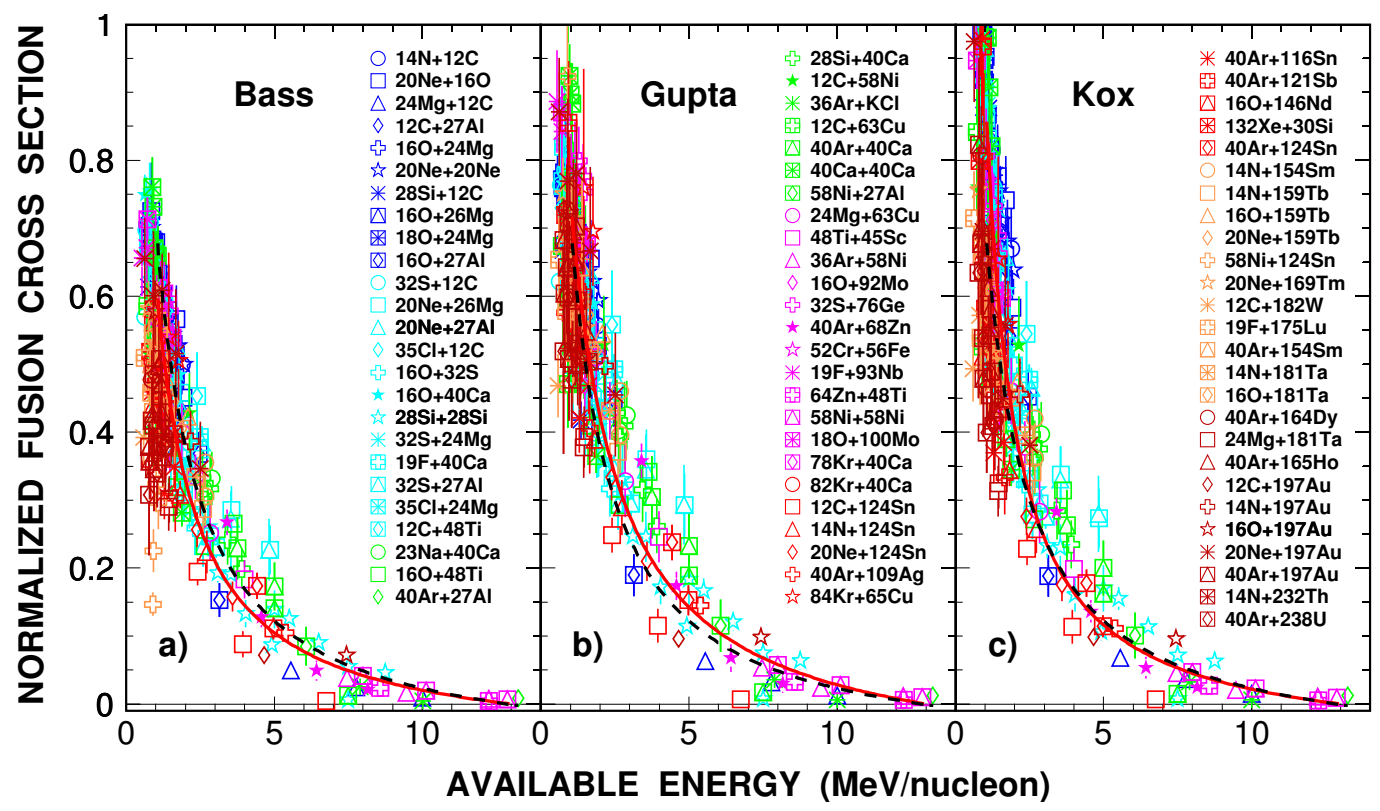

Figure 3. Normalized fusion cross sections $\sigma^{\text {red }}$ plotted as a function of $E_{\text {avail }}$. The $\sigma_{\text {reac }}$ used for normalization is due to a) Bass [8], b) Gupta [9], and c) Kox [10], respectively. The full red curve in each panel is due to a fit with the homographic function (3), whereas the dashed black curve is due to the same kind of fit of panel c) in figure 4.

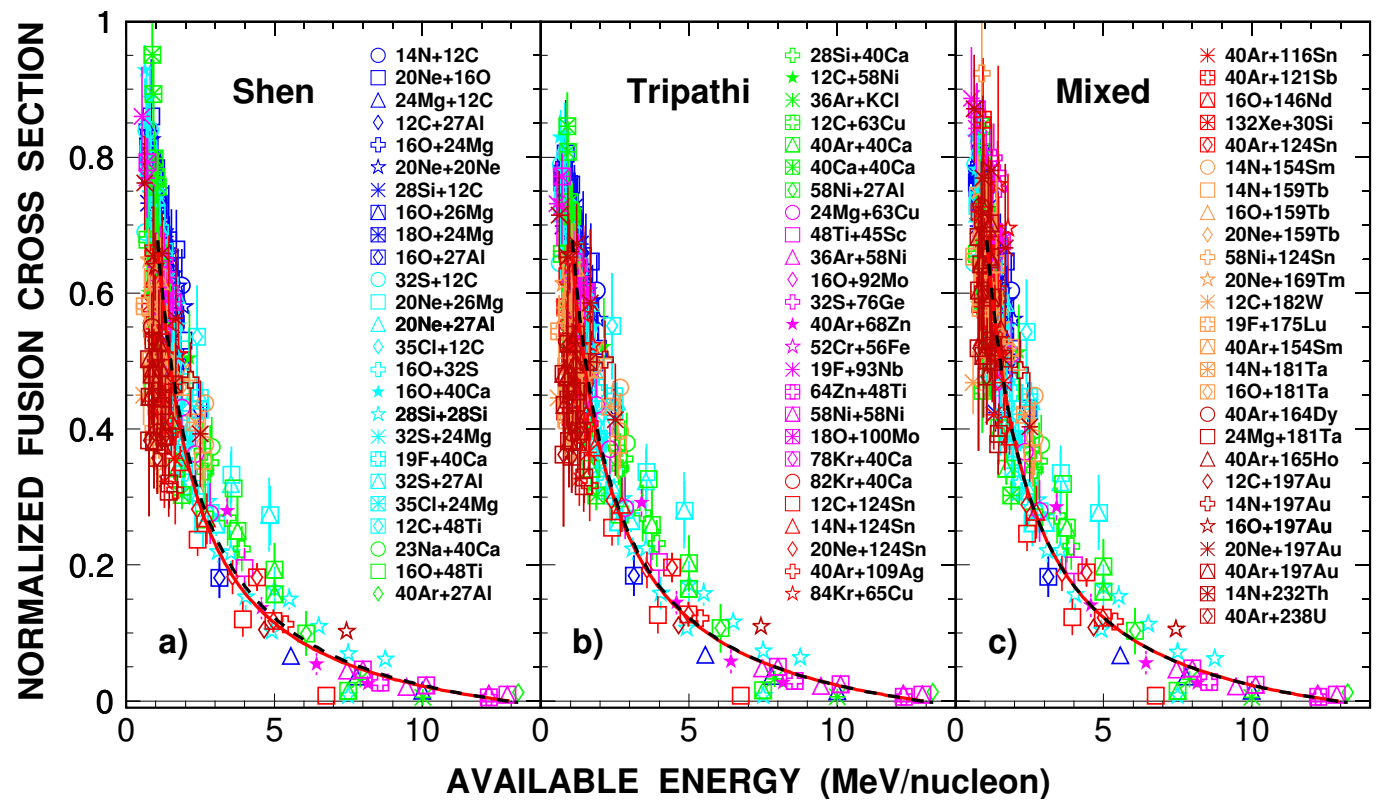

Figure 4. Normalized fusion cross sections $\sigma^{\text {red }}$ plotted as a function of $E_{\text {avail }}$. The $\sigma_{\text {reac }}$ used for normalization is due to a) Shen [11], b) Tripathi [12], and c) the Mixed one (see text for details), respectively. The full red curve in each panel is due to a fit with the homographic function (3), whereas the dashed black curve is due to the fit of panel c).

ures 3 and 4 have been fitted with the three-parameters homographic function (3). The obtained best fit result is displayed by the red full curve for each $\sigma^{\text {red }}$. Interestingly enough, independently of the $\sigma_{\text {reac }}$ chosen for the normalization procedure, the best fit curve gives for the energy of disappearance of the $\mathrm{CF}+\mathrm{IF}$ fusion process the same value: $E_{\text {avail }}=12.98 \pm 0.06 \mathrm{MeV} /$ nucleon. Also, at low energy all the best fit curves overlap. The fit results of different normalization cases disagree by at most 3 to $4 \%$ of $\sigma^{\text {red }}$ and this occurs at intermediate values of $E_{\text {avail }}$. That may be easily inferred by the dashed black curve that is drawn in each panel of figures 3 and 4 and which represents the best fit result in the case of the heuristic (Mixed) $\sigma_{\text {reac }}$. Although the best fit result does not depend strongly on the details of the $\sigma_{\text {reac }}$ parameterization this subject is open to further investigation. However, the homographic functional law (3) seems to be established without any doubt.

\section{Complete fusion cross section}

Only twelve experiments have explicitly been designed to measure both complete and incomplete fusion components 


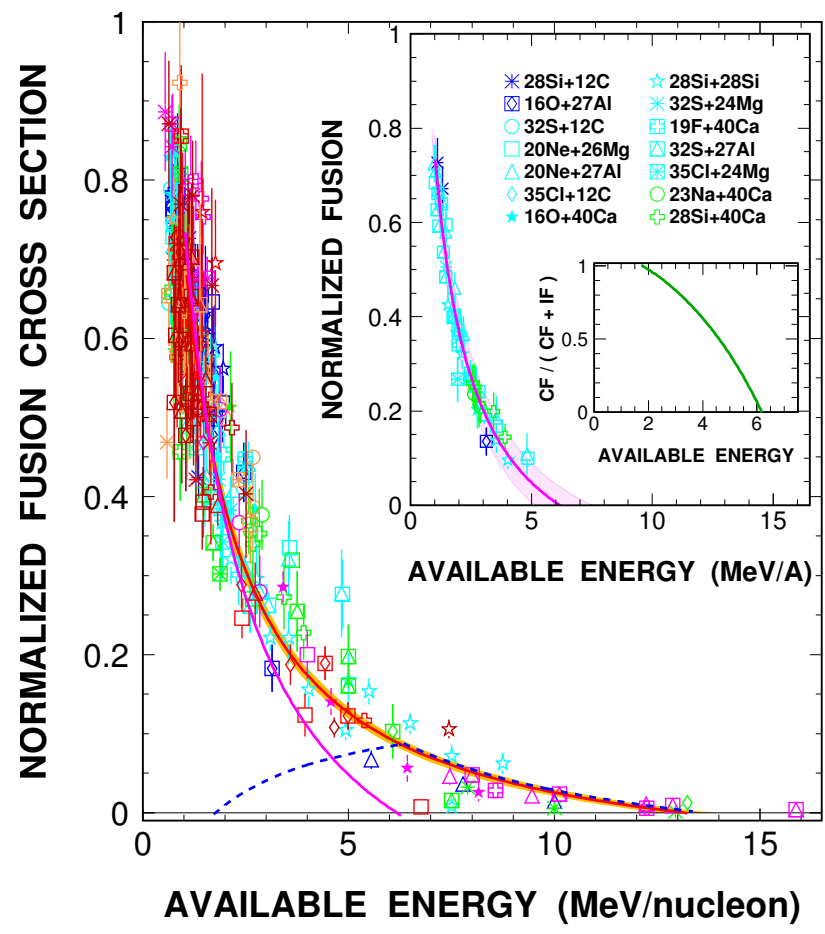

Figure 5. Normalized fusion cross sections $\sigma_{\text {red }}$ for both the complete and incomplete fusion data (main panel, in fact, repeated panel c) of figure 4) and for the complete fusion data (main inset) as a function of $E_{\text {avail }}$. The full violet curve in the inset and the main panel is due to the best fit of the complete fusion data by the homographic function (3). The full red curve in the main panel is due to the same kind of fit to CF+IF data. The orange and light-violet background bands around the best fit curve in the main panel and in the inset, respectively are due to the errors on the fit parameters. The dashed blue curve is the difference of both fusion excitation functions. The inset in the main inset displays the ratio of $\mathrm{CF}$ and $\mathrm{CF}+\mathrm{IF}$ best-fit excitation functions as a function of $E_{\text {avail }}$.

$[5,6]$. These $57 \mathrm{CF} \sigma^{\text {red }}$ data points belonging to the 14 reaction systems and obtained with the heuristic $\sigma_{\text {reac }}$ discussed above are displayed in the main inset of figure 5 as a function of $E_{\text {avail }}$. The same homographic law (3) used in fitting the CF+IF data is here used to obtain the best fit result to the CF data. It is shown by the full violet curve. The fitting code which is used provides an uncertainty on the fit parameters $a, b$, and $c$ [13]. These uncertainties define the light-violet background drawn around the best fit curve. Owing to the rather large experimental error bars and the relatively small number of data points the energy of CF disappearance is not very accurately defined. It reads $E_{\text {avail }}=6.2_{-1.1}^{+1.3} \mathrm{MeV} /$ nucleon. Similarly, $\mathrm{CF}$ data display a stronger dependence on the normalization $\sigma_{\text {reac }}$ used. However, the deduced energy of CF disappearance for each of the $\sigma_{\text {reac }}$ used lays well within the above stated uncertainty limits.

\section{Discussion and conclusions}

In the main panel of figure 5 is repeated panel c) of figure 4, namely $\sigma^{\text {red }}$ for the $\mathrm{CF}+\mathrm{IF}$ data points obtained with the heuristic $\sigma_{\text {reac }}$ displayed as a function of $E_{\text {avail }}$. The orange background drawn around the full red best fit curve is due to the uncertainties of the fit parameters. Much smaller errors of the fit parameters are in accordance with the already discussed stability of the $\mathrm{CF}+\mathrm{IF} \sigma^{\text {red }}$ data. In the main panel is repeated the full violet best fit curve to the $\mathrm{CF}$ data. Making difference of both allows to infer the main properties of the incomplete fusion excitation function: IF process opens around $E_{\text {avail }} \approx 1.5 \mathrm{MeV} /$ nucleon, reaches maximal value at the energy of $\mathrm{CF}$ disappearance $\left(E_{\text {avail }} \approx 6 \mathrm{MeV} /\right.$ nucleon $)$, and vanishes at $E_{\text {avail }} \approx 13$ $\mathrm{MeV} /$ nucleon. An inset in the main inset displays how the best-fit $\mathrm{CF}$ excitation function decreases relatively to the $\mathrm{CF}+\mathrm{IF}$ one, an observable which has been investigated a long time ago by Morgenstern et al. [14].

To summarize, the scrutiny of the existing fusion cross sections well above the reaction barrier allowed us to establish a universal dependence of these data on energy. The established homographic functional description of both the complete and the complete plus incomplete fusion excitation functions is rather stable and the inferred global features of these excitation functions quite weakly depend on the details of the data normalization. Nevertheless, the normalization may be improved if additional high quality measurements on both fusion and reaction cross section would be available.

\section{References}

[1] J.A. Maruhn et al., Comput. Phys. Commun. 185, 2195 (2014)

[2] C. Simenel, Eur. Phys. J. A 48, 152 (2012)

[3] F. Sébille et al., Phys. Rev. C 76, 024603 (2007)

[4] M. Dutra et al., Phys. Rev. C 85, 035201 (2012)

[5] P. Eudes et al., Europhys. Lett. 104, 22001 (2013)

[6] P. Eudes et al., Phys. Rev. C 90, 034609 (2014)

[7] P. Eudes et al., Nucl. Phys. A 930, 131 (2014)

[8] R. Bass, Nuclear Reactions with Heavy Ions (Springer, Berlin, 1980)

[9] S.K. Gupta and S. Kailas, Z. Phys. A 317, 75 (1984)

[10] S. Kox et al., Phys. Rev. C 35, 1678 (1987)

[11] W. Shen et al., Nucl. Phys. A 491, 130 (1989)

[12] R.K. Tripathi, F.A. Cucinotta, and J.W. Wilson, Nucl. Instr. Meth. Phys. Res. B 117, 347 (1996)

[13] F. James and M. Roos, Comput. Phys. Commun. 10, 343 (1975)

[14] H. Morgenstern et al., Phys. Rev. Lett. 52, 1104 (1984) 